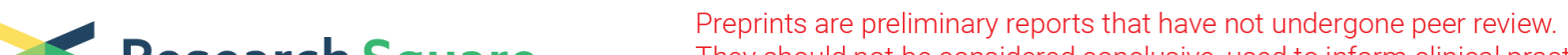 Research Square They should not be considered conclusive, used to inform clinical practice, or referenced by the media as validated information.
}

\section{An Increasing Population of Patients With Oropharyngeal Carcinoma in China is Predicted to Benefit From Proton Beam Therapy in Terms of Cost-effectiveness Consideration}

\section{Guo Li}

Guangzhou Medical University Affiliated Cancer Hospital

Yun-Fei Xia

Sun Yat-sen University Cancer Center

Yi-Xiang Huang

Sun Yat-Sen University

Deniz Okat

Hong Kong University of Science and Technology

Bo Qiu

Sun Yat-sen University Cancer Center

Jerome Doyen

Centre Antoine-Lacassagne

Pierre-Yves Bondiau

Centre Antoine-Lacassagne

Karen Benezery

Centre Antoine-Lacassagne

Jin Gao

University of Science and Technology of China

Chao-Nan Qian ( $\nabla$ qianchn@sysucc.org.cn )

651 Dongfeng East Road, Guangzhou, Guangdong 510060, P. R. China https://orcid.org/0000-00027233-8831

\section{Research article}

Keywords: Oropharyngeal cancer, proton beam therapy, cost-effectiveness analysis, intensity-modulated proton radiation therapy, intensity-modulated photon-radiation therapy, Markov model, China

Posted Date: October 5th, 2020

DOl: https://doi.org/10.21203/rs.3.rs-76204/v1 
License: (c) (i) This work is licensed under a Creative Commons Attribution 4.0 International License. Read Full License

Version of Record: A version of this preprint was published at BMC Cancer on August 21st, 2021. See the published version at https://doi.org/10.1186/s12885-021-08638-2. 


\section{Abstract}

Background: Proton beam therapy (PBT) is a new-emerging cancer treatment in China. The treatment costs are high and not yet covered by Chinese public medical insurance. The advanced form of PBT, intensity-modulated proton radiation therapy (IMPT), has been confirmed to reduce normal tissue complication probability (NTCP) compared with conventional intensity-modulated photon-radiation therapy (IMRT) in patients with oropharyngeal cancer (OPC). This study evaluated the cost-effectiveness of IMPT versus IMRT for OPC patients in China, aiming at guiding proper use of PBT.

Methods: On the basis of published data, a 7-state Markov model was designed for cost-effectiveness analysis, and an evaluation of average level was performed on a base case of 56-year-old under the hypothesis that IMPT could make a $25 \%$ NTCP-reduction concerning to long-term symptomatic dysphagia and xerostomia. Model robustness was examined using probabilistic sensitivity analysis, cohort analysis and tornado diagram. One-way sensitivity analyses were performed to identify costeffective scenarios. IMPT was considered as cost-effective if the incremental cost-effectiveness ratio (ICER) was below the societal willingness-to-pay (WTP) threshold (3 times the gross domestic product per capita / quality-adjusted life year (QALY)).

Results: Compared with IMRT, IMPT could provide an extra 0.724 QALYs at an additional cost of 34,926.6 US dollars (\$), and made an ICER of \$48,229.8/ QALY for the base case. At current WTP level of China $(\$ 30,828 / Q A L Y)$, cost-effective scenarios of IMPT existed in the following independent conditions: $\geq$ 57.3\% NTCP-reduction (IMPT compared with IMRT) in dysphagia and xerostomia; patient age $\leq 38$-yearold; or the cost of IMPT $\leq \$ 37,398.1$. The estimated cost-effective population that benefit from using PBT to treat OPC increased remarkably in the past 10 years with the economic growth, and reached to 559.7 million (about $40.0 \%$ of the China's total population) in the year 2020.

Conclusions: Currently, using PBT to treat OPC could be cost-effective in considerable proportion of China's population. Considering the economic growth, the gradual increment of medical insurance coverage, as well as the proton treatment cost reduction along with more proton facility opening in the near future, it is estimated that PBT would benefit more Chinese OPC patients with respect to costeffectiveness.

\section{Background}

The incidence of oropharyngeal cancer (OPC) has increased in the past 40 years worldwide because of the rising epidemic of human papillomavirus (HPV) infection related to sexual behavior [1]. Traditionally, China has a lower burden of HPV-positive OPC compared to the rest of the world. In the recent decade, the occurrence of OPC in China has showed an upward trend, which is probably due to the behavior changes brought by high pressure lifestyle. As reported, the age-standardized rate of incidence had increased from $2.0 / 100,000$ per year to $2.54 / 100,000$ per year during the year 2003-2012 [2, 3]. 
According to the latest treatment guidelines, radiotherapy/concurrent chemoradiotherapy has been recommended as the mainstream treatment for OPC patients with early/locally advanced stage, whether HPV-positive or HPV-negative [4, 5]. Owing to the anatomic location and a high required radical dose of this tumor type, irradiation-induced dysphagia and xerostomia become the most common late toxicities in OPC survivors. Even with the best photon irradiation technique, intensity-modulated photon-radiation therapy (IMRT), symptomatic dysphagia and xerostomia (grade 2-4) occurred with the incidences as high as $15-23 \%$ and $32-48 \%$; and were confirmed as independent negative influential factors impacting OPC patient's long-term life quality [6-9].

Proton beam therapy (PBT) is a new-emerging particle irradiation technique with superior energy absorption distribution afforded by protons' "Bragg peaks" to protect normal tissues. In recent comparative studies, intensity-modulated proton radiation therapy (IMPT), the advanced form of PBT, was found to lower irradiation dose of normal tissues including the parotid, larynx, oral cavity, and pharyngeal constrictor muscle in OPC patients, resulting in the normal tissue complication probability (NTCP) concerning to long-term symptomatic dysphagia and xerostomia reduced by approximate $25 \%$ on average, in comparison to that of IMRT [10-13]. In previous PBT decision-making studies for OPC, such advantages of IMPT over IMRT in reducing dysphagia and xerostomia had been applied as the pivotal factor determining whether the OPC patient was appropriate to undergo PBT [14, 15].

Nevertheless, the therapeutic benefits should still be carefully weighed against the proton's much higher treatment cost, which is 3.2 to 4.8 times that of IMRT [16]. At the mainland of China, there is only 1 operational proton center at present, the government has planned to authorize another 16 licenses for operating proton center in the major cities by the year 2020 [17]. The high treatment costs of this newemerging cancer treatment are not yet covered by Chinese public medical insurance. Cost-effectiveness analysis (CEA) is urgently needed in guiding proper clinical decision of PBT when this treatment would become more available in the near future $[18,19]$. Till present, no CEA studies was conducted to evaluate the cost-effectiveness of PBT for OPC patients in China.

On the basis of published data, we designed a 7-state Markov model to track the natural development of disease and late toxicities and simultaneously evaluate the relevant cost and effectiveness of IMPT versus IMRT in a long-term period for Chinese OPC patients. The analyses of this study aimed to identify the cost-effective scenarios and estimate the potential population that benefit from using PBT to treat OPC in China.

\section{Methods}

\section{Model design}

TreeAge Pro 2018 software (Williamstown, MA) was used for model building and analysis. A decision tree combined with two 7-state Markov models were designed to evaluate the cost-effectiveness of IMPT in comparison to that of IMRT for newly diagnosed non-metastatic OPC patients. CEA modelling was based 
on two hypotheses: (1) the advantage of IMPT over IMRT would be its ability in reducing symptomatic dysphagia and xerostomia (Radiation Therapy Oncology Group, RTOG grade 2-4), defined as "NTCPreduction" in this study, and NTCP-reduction (\%) = [(NTCP after IMRT - NTCP after IMPT) / NTCP after IMRT $]$ * $100 \%$; (2) all the dysphagia and xerostomia would occur within the first year after radiotherapy and late toxicities would be irreversible if occurred $[20,21]$.

Transition states of the Markov model were illustrated in Fig. 1. The states of "alive with cancer", "no cancer" (including 4 sub-states of "dysphagia", "xerostomia", "dysphagia and xerostomia" and "no complication") and "death" (including "cancer death" or "other death") were used to simulate the natural process of disease and late toxicities for OPC patients after radiotherapy. A 1-year cycle length was used, and the Markov models were cycled from 1 year after radiotherapy until the estimated generalized Chinese life expectancy in 2020 (77-year-old) to evaluate the cost-effectiveness of a lifetime after radiotherapy [22]. The risk of natural non-cancer caused death was calculated based on the 2016 Life Tables of United States [23].

\section{Base case set-up}

We used a base case of 56-year-old (median age of this cancer type in China) to represent the average Chinese OPC patients [24]. Disease probabilities in the model were calibrated according to the overall survival (OS) and disease-free survival (DFS) data for OPC patients, reported by F De Felice et al. [25]. The 2 compared treatment strategies were similar except for the irradiation technique (IMPT versus IMRT), which included a radical radiotherapy and 3 cycles of concurrent chemotherapy. The NTCPs of dysphagia and xerostomia after IMRT were set according to the long-term follow-up results of OPC patients after IMRT reported by Bird T et al. and Al-Mamgani A et al., in which the probabilities of dysphagia and xerostomia ( $\geq$ grade 2 ) were 0.19 and 0.33 respectively $[5,6]$. On the basis of the published data, we assumed that IMPT could make a $25 \%$ NTCP-reduction in both dysphagia and xerostomia compared with IMRT for average OPC patients $[12,13]$. Other clinical outcomes, including the survival rates, were assumed to be identical between the two strategies.

\section{Cost and utilities}

The costs of treatment were calculated based on casual clinical prescriptions to reflect similar costs as that of daily practice in a Chinese hospital. All costs were adjusted to US dollars (\$), using a Sino-US exchange rate of $\$ 1=6.93 \mathrm{RMB}$ (January 23,2020 ). The cost of IMPT and IMRT were estimated as being $\$ 50,000$ and $\$ 12,000$ respectively for simulating a 32 -fraction to a total dose of $70 \mathrm{~Gy}$. The cost of concurrent chemotherapy was assumed as $\$ 5,000$ (simulating 3 cycles of $80100 \mathrm{mg} / \mathrm{m}^{2}$ cisplatin bolus injection delivered on day-1, -21 and -42 of the radiotherapy). The follow-up cost per year was assumed as $\$ 1,000$ (including hematologic and biochemistry profiles, nasopharyngeal fiberoptic endoscope examination, magnetic resonance imaging of head and neck, chest radiography and abdominal ultrasonography); the cost of palliative therapy per year was assumed as $\$ 5,000$ (simulating 8 cycles of oral palliative chemotherapy with 5-fluorouracil). The treatment cost for dysphagia per year was 
estimated to be $\$ 3,000$; simulating long-term use of nasal feeding tube or percutaneous gastrostomy tube. The treatment cost for xerostomia per year was estimated to be $\$ 2,000$; simulating the consumption of traditional Chinese medicine or artificial saliva. For patients in the "no cancer" state, the incremental cost per year was the follow-up cost \pm the treatment cost for dysphagia and/or xerostomia. For patients in the "alive with cancer" state (recurrence, metastasis, or residue), the incremental cost per year included the follow-up cost and the cost of palliative chemotherapy.

The utilities were adjusted to QALYs using health state utility values (HSUV) derived from previously published studies for head and neck cancer. HSUV can be interpreted as the strength of preference for a given health state on a cardinal scale anchored at 0 ('death') and 1 ('full health'). In this model, the HSUV of "alive with cancer" was assumed as 0.57 to represent a progressive disease with the disutility caused by anticancer treatment [26]. The HSUVs for the states of "dysphagia", "xerostomia" and "dysphagia and xerostomia" ( $\geq$ grade 2 ) were standardized as $0.803,0.846$ and 0.763 , according to the report of Ramaekers et al. [9]. The HSUVs for the states of "no complication" and "death" were set as 1 and 0. Costs and quality-adjusted life-years (QALYs) were discounted at an annual rate of $3 \%$ [27].

\section{Outcomes measurement}

OS was defined as time interval between the end of the radiotherapy and death from any cause. DFS was defined as the time interval between the end of the radiotherapy and first cancer progression or death from any cause. The outcome measure of the model was the incremental cost-effectiveness ratio (ICER) which represented the ratio of the difference in costs to the difference in effectiveness (incremental cost / incremental effectiveness) between IMPT and IMRT. A strategy is deemed cost-effective by comparing the ICER with an established societal willingness-to-pay (WTP). According to the World Health Organization guidelines, a strategy is defined as cost-effective if the ICER value is below 3 times the gross domestic product (GDP) per capita. In this study, \$30,828 / QALY (3 times the Chinese GDP per capita in 2019) was applied as the current WTP level of China [28, 29].

\section{Sensitivity analysis and Monte Carlo simulation}

Probabilistic sensitivity analysis was applied to illustrate the robustness of the model in light of a joint uncertainty for model parameters by running over 50,000 iteration trials, and the $90 \%$ confidence interval of the model parameters were identified. Tornado diagram was used to evaluate the influences of the parameters on the ICER over the variation of their $90 \%$ confidence interval. One-way sensitivity analyses were performed to identify the cost-effective scenarios of IMPT. Monte Carlo simulation (50,000 trials) was applied to show trials distribution of the two strategies and determine which would be the recommended strategy from the perspective of net benefit.

\section{Estimation of cost-effective population}

The population with a WTP level beyond the ICER value was estimated as cost-effective population in China in this study. All the 397 administrative regions (cities) at the mainland of China were ranked according to their GDP per capita level. The forefront regions in the ranking, with accumulated total GDP 
and total population that corresponded an average GDP per capita that made a WTP threshold value (3 times the GDP per capita) beyond the ICER value, were delineated as the cost-effective regions; and the total population in these forefront regions were estimated as the cost-effective population in China. All GDP and population data were collected from the official website of Chinese National Bureau of Statistics [30].

\section{Results}

\section{Model robustness verification}

The model input information and probabilistic sensitivity analyses for the model parameters are summarized in Table 1. The model robustness verification was performed using the base case set-up. Markov cohort analyses for both IMPT strategy and IMRT strategy are shown in Additional file 1: Figure S1. The CEA model predicted the 1- ,3-, 5- and 10 -year OS rates of $90.0 \%, 85.5 \%, 80.0 \%$ and $68.7 \%$; and the 1- ,3-, 5- and 10-year DFS rates of $85.0 \%, 78.8 \%, 72.9 \%$ and $67.1 \%$ for average OPC patients. Such survival data were very similar with the previous outcomes reported by F De Felice et al. [25] (detailed in Additional file 2: Figure S2).

The tornado diagram identified the top 5 parameters influencing the ICER as the NTCP of dysphagia after IMPT, the cost of IMPT, the NTCP of xerostomia after IMPT, the NTCP of dysphagia after IMRT and the NTCP of xerostomia after IMRT; and the other parameters had only a minor impact on the ICER (Fig. 2).

\section{Cost-effectiveness of the base case}

By model calculation, IMPT (compared with IMRT) provided the base case an additional 0.724 QALYs at an additional cost of $\$ 34,926.6$, and the ICER was $\$ 48,229.8 /$ QALY. Thus, IMPT was not cost-effective for the base case at the current WTP level of China $(\$ 30,828 / Q A L Y)$. IMPT was the recommended strategy in $34.0 \%$ of trials from the perspective of net benefit in Monte Carlo simulations, as showed in Additional file

\section{3: Figure S3.}

\section{Cost-effective scenarios of proton beam therapy}

One-way sensitivity analyses identified the potential cost-effective scenarios of IMPT with base case setup, using 3 different WTP thresholds (\$30,828 / QALY, $\$ 50,000$ / QALY and $\$ 100,000 /$ QALY) (Table 2). At the current WTP level of China $(\$ 30,828$ / QALY), the cost-effective scenarios of IMPT existed in the following independent conditions: $\geq 57.3 \%$ NTCP-reduction (IMPT compared with IMRT) in dysphagia and xerostomia; patient age $\leq 38$-year-old; or the cost of IMPT $\leq \$ 37,398.1$.

\section{Cost-effective population in China benefit from proton beam therapy}

The cost-effective population that benefit from using PBT to treat OPC in China was estimated for the years from 2010 to 2020, summarized in Additional file 4: Table S1. The estimated cost-effective population increased remarkably in the past 10 years with the growth of GDP per capita in China; and 
such population reached to 559.7 million, about $40.0 \%$ of the China's total population in the year 2020, as shown in Fig. 3 and Additional file 5: Table S2.

\section{Discussion}

In this study, a reliable and robust 7-state Markov model was designed to evaluate the cost-effectiveness of IMPT versus IMRT for OPC, a fast-growing malignancy in China. On the basis of published data, the cost-effective scenarios of IMPT at the current WTP level of China were identified as the following independent conditions: $\geq 57.3 \%$ NTCP-reduction (IMPT compared with IMRT) in dysphagia and xerostomia; patient age $\leq 38$-year-old; or the cost of IMPT $\leq \$ 37,398.1$. The cost-effective population in China that benefit from using PBT to treat OPC increased remarkably in the past 10 years with the growth of GDP per capita. It was estimated that such cost-effective population would be about $40.0 \%$ of the China's total population in the year 2020.

Cost-effectiveness of PBT has been poorly evaluated worldwide, and has been referred as the proton's "economic controversy" [18, 31]. The only documented CEA study of PBT for OPC patients was reported by Sher DJ et al., a 6-state Markov model was designed for a 65-year-old OPC patient based on a hypothesis that IMPT could make a $25 \%$ reduction of xerostomia, dysgeusia and the need for gastrostomy tube, with the model set-up reflecting the practice in American hospitals [32]. But the 6-state Markov model in that study was found not applicable for Chinese OPC patients in certain regards, such as Chinese patients' unwillingness to undergo invasive surgery like percutaneous gastrostomy tube for treating the eating difficulty, and the extensive use of traditional Chinese medicine in dealing with xerostomia.

Markov model design is a key step of CEA modelling for PBT. In our previous CEA modelling for paranasal sinus and nasal cavity cancer, a reliable 3-state Markov model was designed to simulate the tumor development and evaluate the cost-effectiveness of IMPT in comparison to IMRT in terms of tumor control improvement [33]. Unlike paranasal sinus and nasal cavity cancer, the advantages of IMPT over IMRT for OPC patients lied in reducing late toxicities while not in improving tumor control or survival rates $[34,35]$. In this CEA modelling for OPC, we also used 3 main states including "alive with cancer", "no cancer" and "death" to simulate the tumor development of OPC, but the survival probabilities were set identical between IMPT strategy and IMRT strategy. The model-predicted OS and DFS were found correspond to the previous long-term survival outcomes [25], which demonstrated that the CEA modelling did abide with the natural disease process of OPC.

To evaluate long-term differences in effectiveness and cost between the two strategies, the state of "no cancer", referring to OPC survivor after radiotherapy, was further divided into 4 sub-states of "dysphagia", "xerostomia", "dysphagia and xerostomia" and "no complication", the initial state probabilities of the 4 sub-states were set according to the NTCPs of dysphagia and xerostomia. For each cycle, the HSUVs for states of "dysphagia", "xerostomia", "dysphagia and xerostomia" and "no complication" were set as $0.803,0.846,0.763$ and 1 , respectively; meanwhile, the annual costs for dealing with these toxicities were 
added as the accumulated incremental costs. Therefore, NTCP-reductions of dysphagia and xerostomia (IMPT compared with IMRT) became the original motivities of differences in effectiveness and cost between the two strategies. The robustness of this model design was confirmed by tornado diagram analysis, which demonstrated that only the NTCPs and the cost of IMPT had major impacts on ICER value.

In this study, the cost-effective scenarios of PBT were identified as 3 independent conditions for Chinese OPC patients: $\geq 57.3 \%$ NTCP-reduction, patient age $\leq 38$-year-old, or the cost of IMPT $\leq \$ 37,398.1$. In previous PBT decision-making studies for OPC patients, the superiority of IMPT over IMRT in reducing dysphagia and xerostomia (NTCP-reductions) has been applied as the determining factor for treatment decision making between IMPT and IMRT, and NTCP models were applied to estimate NTCP-reductions using dosimetric information in IMPT and IMRT treatment plans, such as the approaches developed by Langendijk JA et al. and Brodin et al. [14, 15]. However, the threshold of NTCP-reduction, above which an OPC patient could be considered as appropriate for PBT, had not be defined in the previous studies. Here, we first defined a threshold for NTCP-reduction based on the criterion of cost-effectiveness. In our CEA modelling, we also found that younger patient who had a longer survival time could benefit more from PBT from the cost-effectiveness consideration. Thus, patient age should be another main consideration in the clinical decision making of prescribing PBT. In addition, proton treatment cost was identified as one of the major influential factors to the cost-effectiveness, and the current proton treatment cost was found high at current WTP level of China. But we estimated that such treatment cost will probably be reduced in the near future due to the market competition and technology upgrade along with more proton centers open in China [36].

Of note, at current stage without public medical insurance coverage, the level of ability-to-pay (WTP level) should also be weighed in clinical decision of using PBT. Regional difference of WTP level in China is obvious. For example, the GDP per capita in Shenzhen City of China (the second highest) are as high as $\$ 28,931$ in the year 2019, corresponded a WTP of $\$ 86,793 /$ QALY (2.8 times the current WTP of China). In order to evaluate the acceptance of using PBT to treat OPC in whole population of China, we delineated the population of a higher level of GDP level that corresponded a WTP level beyond the ICER value $(\$ 48,229.8 / Q A L Y)$ as an estimated cost-effective population in this study. Such cost-effective population was found increased remarkably in the 10 past years with the growth of GDP per capita, and reached to 559.7 million (about $40.0 \%$ of the China's total population) in the year 2020. This finding indicated the great market potential and future trend of PBT in Chinese society, even though such estimation might not be accurate due to the uneven distribution of social wealth.

There are two main limitations in this study. First, our CEA modelling was hypothesis-based. We assumed that all the symptomatic dysphagia and xerostomia would occur within the first year after radiotherapy and late toxicities would be irreversible if occurred. This assumption was based on the previously observed studies, which showed that the changes of two late toxicities would be tiny and negligible after 1 year [37, 38]. The Markov models were cycled from 1 year after radiotherapy, and the potential differences in irradiation-induced adverse events within the first year after radiotherapy, including the 
acute toxicities and hospital admission rates, were not taken into account in CEA. Hence, these hypothesis-related problems may hamper the interpretation of the results. Second, the CEA modelling in this study was performed with the base case set-up (a 25\% NTCP-reduction, 56-year-old, and the current WTP of China) to represent Chinese OPC patients of an average level. However, the clinical decision making of prescribing PBT to a specific OPC patient should base on CEA modelling with individualized set-up, which enables to take patient's age, tumor stage and economic situation into account. So, the next step, we plan to conduct individualized CEA modelling for treatment decision making (IMPT versus IMRT).

\section{Conclusions}

On the basis of published data and hypothesis-based CEA modelling, the results in this study demonstrated that the cost-effective scenarios of PBT did exist in Chinese OPC patients; and using PBT to treat OPC could be cost-effective in considerable proportion of China's population at current stage. Considering future changes including the economic growth, the gradual increment of medical insurance coverage and the proton treatment cost reduction, it is predicted that PBT would benefit more Chinese OPC patients with respect to cost-effectiveness.

\section{Abbreviations}

PBT: Proton beam therapy; IMPT: Intensity-modulated proton radiation therapy; IMRT: Intensitymodulated photon-radiation therapy; OPC: Oropharyngeal cancer; NTCP: Normal tissue complication probability; PSA: Probabilistic sensitivity analysis; ICER: Incremental cost-effectiveness ratio; WTP: Willingness-to-pay; HPV: Human papillomavirus; CEA: Cost-effectiveness analysis; RTOG: Radiation Therapy Oncology Group; OS: Overall survival; DFS: Disease-free survival; GDP: Gross domestic product; \$: US dollars; QALY: Quality-adjusted life year; SPHIC: Shanghai proton and heavy lon Center; SYSUCC: Sun Yat-sen University Cancer Center; HSUV: Health state utility value.

\section{Declarations}

\section{Ethics approval and consent to participate}

Not applicable.

\section{Consent to publication}

Not applicable.

\section{Availability of data and materials}

The datasets used and analyzed during the current study are available from the corresponding author on reasonable request. 


\section{Competing interests}

The authors declare that they have no competing interests.

\section{Funding}

This work was supported by grants to CNQ from the National Natural Science Foundation of China (No. 81872384, No. 81672872, and No. 81472386), the Provincial Natural Science Foundation of Guangdong, China (No. 2016A030311011), a research program from the Sun Yat-sen University (No. 8400018843409); and by grants to YFX from the National Nature Science Foundation of China (No. 81872464) and the Science Development Program of Guangzhou.

\section{Authors' Contributions}

CNQ and GL were responsible for the concept and design of the analysis. JG, CNQ and YXH collected data for analysis. GL, DO, YXH, PYB and BQ performed the analysis and interpretation of results. GL and CNQ prepared a first draft of the manuscript. JD, KB, BQ and YFX provided critical revision to the first and subsequent drafts of the manuscript. All authors contributed to and have reviewed and approved the final version of the manuscript. CNQ is the guarantor of the manuscript.

\section{Acknowledgments}

Not applicable.

\section{Authors' Information}

Details in authors' names and affiliations in Title Page part.

\section{References}

1. Bhatia A, Burtness B. Human papillomavirus pssociated oropharyngeal cancer: defining risk groups and clinical trials. J Clin Oncol. 2015;33:3243-3250.

2. Zhang LW, Li J, Cong X, Hu XS, Li D, Wu LL, et al. Incidence and mortality trends in oral and oropharyngeal cancers in China, 2005-2013. Cancer Epidemiol. 2018;57:120-126.

3. Liu J, Yang XL, Zhang SW, Zhu LP, Chen WQ. Incidence, mortality, and temporal patterns of oropharyngealcancer in China: a population-based study. Cancer Commun (Lond). 2018;38:75.

4. Grégoire V, Nicolai P. Choosing surgery or radiotherapy for oropharyngeal squamous cell carcinoma: is the issue definitely settled? Lancet Oncol. 2019;20:1328-1329.

5. Townsend M, DeWees T, Gross J, Daly M, Gay H, Thorstad W, et al. Timing of postoperative radiotherapy in surgically treated HPV-positive oropharyngeal squamous cell carcinoma. Otolaryngol Head Neck Surg. 2019;161:297-306. 
6. Bird T, De Felice F, Michaelidou A, Thavaraj S, Jeannon JP, Lyons A, et al. Outcomes of intensitymodulated radiotherapy as primary treatment for oropharyngeal squamous cell carcinoma - a European single institution analysis. Clin Otolaryngol. 2017;42:115-122.

7. Al-Mamgani A, van Rooij P, Verduijn GM, Mehilal R, Kerrebijn JD, Levendag PC. The impact of treatment modality and radiation technique on outcomes and toxicity of patients with locally advanced oropharyngeal cancer. Laryngoscope. 2013;123:386-393.

8. Goepfert RP, Lewin JS, Barrow MP, Gunn GB, Fuller CD, Beadle BM, et al. Long-term, prospective performance of the MD Anderson dysphagia inventory in "Low-Intermediate Risk" oropharyngeal carcinoma after intensity modulated radiation therapy. Int J Radiat Oncol Biol Phys. 2017;97:700708.

9. Sapir E, Tao Y, Feng F, Samuels S, El Naqa I, Murdoch-Kinch CA, et al. Predictors of dysgeusia in patients with oropharyngeal cancer treated with chemotherapy and intensity modulated radiation therapy. Int J Radiat Oncol Biol Phys. 2016;96:354-361.

10. Ramaekers BL, Joore MA, Grutters JP, van den Ende P, Jong Jd, Houben R, et al. The impact of late treatment-toxicity on generic health-related quality of life in head and neck cancer patients after radiotherapy. Oral Oncol 2011;47:768-774.

11. Apinorasethkul O, Kirk M, Teo K, Swisher-McClure S, Lukens JN, Lin A. Pencil beam scanning proton therapy vs rotational arc radiation therapy: A treatment planning comparison for postoperative oropharyngeal cancer. Med Dosim. 2017;42:7-11.

12. van de Water TA, Lomax AJ, Bijl HP, de Jong ME, Schilstra C, Hug EB, et al. Potential benefits of scanned intensity-modulated proton therapy versus advanced photon therapy with regard to sparing of the salivary glands in oropharyngeal cancer. Int J Radiat Oncol Biol Phys. 2011;79:1216-1224.

13. Meijer TWH, Scandurra D, Langendijk JA. Reduced radiation-induced toxicity by using proton therapy for the treatment of oropharyngeal cancer. Br J Radiol. 2020;93:20190955.

14. Sio TT, Lin HK, Shi Q, Gunn GB, Cleeland CS, Lee JJ, et al. Intensity modulated proton therapy versus intensity modulated photon radiation therapy for oropharyngeal cancer: First comparative results of patient-reported outcomes. Int J Radiat Oncol Biol Phys. 2016;95:1107-1114.

15. Langendijk JA, Lambin P, De Ruysscher D, Widder J, Bos M, Verheij M. Selection of patients for radiotherapy with protons aiming at reduction of side effects: The model-based approach. Radiother Oncol. 2013;107:267-273.

16. Brodin NP, Kabarriti R, Pankuch M, Schechter CB, Gondi V, Kalnicki S, et al. A quantitative clinical decision-support strategy identifying which patients with oropharyngeal head and neck cancer may benefit the most from proton radiation therapy. Int J Radiat Oncol Biol Phys. 2019;104:540-552.

17. Peeters A, Grutters JP, Pijls-Johannesma M, Reimoser S, De Ruysscher D, Severens JL, et al. How costly is particle therapy? Cost analysis of external beam radiotherapy with carbon-ions, protons and Radiother Oncol. 2010;95:45-53.

18. The notice of the National Health Commission on adjusting the configuration plan of large-scale medical equipment for 2018-2020. National Health Commission of the People's Republic of China. 
2020.

http://www.nhc.gov.cn/caiwusi/s10743/202007/c710ab96137846928ba6154135e085d0.shtml. Accessed September 10, 2020.

19. Lievens Y, Pijls-Johannesma M. Health economic controversy and cost-effectiveness of proton therapy. Semin Radiat Oncol. 2013;23:134-141.

20. Russell LB, Gold MR, Siegel JE, Daniels N, Weinstein MC. The role of cost-effectiveness analysis in health and medicine. Panel on cost-effectiveness in Health and Medicine. JAMA. 1996;276:11721177.

21. Houweling AC, Philippens ME, Dijkema T, et al. A Comparison of dose-response models for the parotid gland in a large group of head-and-neck cancer patients. Int J Radiat Oncol Biol Phys 2010; 76:1259-1265.

22. Christianen ME, Schilstra C, Beetz I, et al. Predictive modelling for swallowing dysfunction after primary (chemo)radiation: results of a prospective observational study. Radiother Oncol 2012;105:107-114.

23. Wang HM. Attaching importance to health of elderly population and promoting national healthy ageing actively in China. Zhonghua Liu Xing Bing Xue Za Zhi 2019;40:259-265.

24. Arias E, Xu J, Kochanek KD. United States Life Tables, 2016. Natl Vital Stat Rep. 2019;68:1-66.

25. Yong-xia Zhang , Bin Zhang, Li Gao, Zhen-gang Xu, Ping-zhang Tang. Clinical analysis of 318 cases of oropharyngeal squamous cell carcinoma. Zhonghua Er Bi Yan Hou Tou Jing Wai Ke Za Zhi. 2013;48:398-404.

26. De Felice F, Bird T, Michaelidou A, Thavaraj S, Odell E, et al. Radical (chemo)radiotherapy in oropharyngeal squamous cell carcinoma: Comparison of TNM 7th and 8th staging systems. Radiother Oncol. 2020;145:146-153.

27. de Almeida JR, Villanueva NL, Moskowitz AJ, Miles BA, Teng MS, Sikora A, et al. Preferences and utilities for health states after treatment for oropharyngeal cancer: Transoral robotic surgery versus definitive (chemo)radiotherapy. Head Neck. 2014;36:923-933.

28. Siegel JE, Weinstein MC, Russell LB, Gold MR. Recommendations for reporting cost-effectiveness analyses. panel on cost-effectiveness in health and medicine. JAMA. 1996;276:1339-1341.

29. Statistical communiqué of the people's republic of China on the 2019 national economic and social development. National Bureau of Statistics of China. stats.gov.cn/english/PressRelease/202002/t20200228_1728917.html. Accessed September 10, 2020 .

30. Murray CJ, Evans DB, Acharya A, Baltussen RM. Development of WHO guidelines on generalized cost-effectiveness analysis. Health Econ. 2000;9:235-51.

31. National Bureau of Statistics of China. http://www.stats.gov.cn/. Accessed September 10, 2020.

32. Verma V, Mishra MV, Mehta MP. A systematic review of the cost and cost effectiveness studies of proton radiotherapy. Cancer. 2016;122:1483-1501. 
33. Sher DJ, Tishler RB, Pham NL, Punglia RS. Cost-effectiveness analysis of intensity modulated radiation therapy versus proton therapy for oropharyngeal squamous cell carcinoma. Int $\mathrm{J}$ Radiat Oncol Biol Phys. 2018;101:875-882.

34. Li G, Qiu B, Huang YX, Doyen J, Bondiau PY, Benezery K, et al.

35. Cost-effectiveness analysis of proton beam therapy for treatment decision making in paranasal sinus and nasal cavity cancers in China. BMC Cancer. 2020;26;20:599.

36. Gunn GB, Blanchard P, Garden AS, Zhu XR, Fuller CD, Mohamed AS, et al. Clinical outcomes and patterns of disease recurrence after intensity modulated proton therapy for oropharyngeal squamous carcinoma. Int J Radiat Oncol Biol Phys. 2016 May 1;95(1):360-367.

37. Aljabab S, Liu A, Wong T, Liao JJ, Laramore GE, Parvathaneni U. Proton therapy for locally advanced oropharyngeal cancer: Initial clinical experience at the university of Washington. Int J Part Ther. 2020;6:1-12.

38. Kerstiens J, Johnstone GP, Johnstone PAS. Proton facility economics: Single-room centers. J Am Coll Radiol. 2018;15:1704-1708.

39. Bagley AF, Ye R, Garden AS, Gunn GB, Rosenthal DI, Fuller CD, et al. Xerostomia-related quality of life for patients with oropharyngeal carcinoma treated with proton therapy. Radiother Oncol. 2020;142:133-139.

40. Grant SR, Hutcheson KA, Ye R, Garden AS, Morrison WH, Rosenthal DI, et al. Prospective longitudinal patient-reported outcomes of swallowing following intensity modulated proton therapy for oropharyngeal cancer. Radiother Oncol. 2020;148:133-139.

\section{Tables}

Table 1 Model information and probabilistic sensitivity analysis for the parameters 


\begin{tabular}{|c|c|c|c|c|}
\hline Parameters & $\begin{array}{l}\text { Input } \\
\text { information }\end{array}$ & $90 \% \mathrm{Cl}$ in $\mathrm{PSA}^{\mathrm{a}}$ & Distribution ${ }^{\mathrm{b}}$ & Source \\
\hline Target Cancer & OPC & & & \\
\hline $\begin{array}{l}\text { Evaluated treatment } \\
\text { strategies }\end{array}$ & $\begin{array}{l}\text { IMPT vs. } \\
\text { IMRT }\end{array}$ & & & \\
\hline \multicolumn{5}{|l|}{ Base case set-up } \\
\hline Patient age ${ }^{c}$ & 56-year-old ${ }^{d}$ & & & \\
\hline \multicolumn{5}{|l|}{ Disease probabilities } \\
\hline DFS (1-year) & 0.85 & & & $\begin{array}{l}\text { F De Felice et al. } \\
\text { [25] }\end{array}$ \\
\hline OS (1-year) & 0.9 & & & $\begin{array}{l}\text { F De Felice et al. } \\
{[25]}\end{array}$ \\
\hline \multirow[t]{2}{*}{$\begin{array}{l}\text { "no cancer" to "alive with } \\
\text { cancer" }\end{array}$} & $\begin{array}{l}0.03\left(2^{\text {nd }}-5^{\text {rd }}\right. \\
\text { year })\end{array}$ & & & $\begin{array}{l}\text { F De Felice et al. } \\
\text { [25] }\end{array}$ \\
\hline & $\begin{array}{l}0\left(6^{\text {th }}-10^{\text {th }}\right. \\
\text { year })\end{array}$ & & & \\
\hline $\begin{array}{l}\text { "alive with cancer" to "cancer } \\
\text { death" }\end{array}$ & 0.3 & & & $\begin{array}{l}\text { F De Felice et al. } \\
\text { [25] }\end{array}$ \\
\hline \multicolumn{5}{|l|}{$\begin{array}{l}\text { Long-term toxicities } \\
\text { probabilities }\end{array}$} \\
\hline $\begin{array}{l}\text { NTCP of dysphagia after } \\
\text { IMRT }\end{array}$ & 0.19 & $0.129-0.256$ & Beta & Bird T et al. [5] \\
\hline $\begin{array}{l}\text { NTCP of xerostomia after } \\
\text { IMRT }\end{array}$ & 0.33 & $0.266-0.394$ & Beta & $\begin{array}{l}\text { Al-Mamgani A et } \\
\text { al. [6] }\end{array}$ \\
\hline $\begin{array}{l}\text { NTCP of dysphagia after } \\
\text { IMPT }\end{array}$ & 0.143 & $0.0369-0.276$ & Beta & $\begin{array}{l}\text { Meijer TWH et } \\
\text { al. [12] }\end{array}$ \\
\hline $\begin{array}{l}\text { NTCP of xerostomia after } \\
\text { IMPT }\end{array}$ & 0.248 & $0.128-0.377$ & Beta & $\begin{array}{l}\text { Meijer TWH et } \\
\text { al. [12] }\end{array}$ \\
\hline \multicolumn{5}{|l|}{ Utilities (QALYs) } \\
\hline No complication & 1 & & & \\
\hline Dysphagia & 0.803 & $0.671-0.918$ & Beta & $\begin{array}{l}\text { Ramaekers BL } \\
\text { et al. [9] }\end{array}$ \\
\hline Xerostomia & 0.846 & $0.714-0.955$ & Beta & $\begin{array}{l}\text { Ramaekers BL } \\
\text { et al. [9] }\end{array}$ \\
\hline Dysphagia and xerostomia & 0.763 & $0.631-0.881$ & Beta & $\begin{array}{l}\text { Ramaekers BL } \\
\text { et al. [9] }\end{array}$ \\
\hline
\end{tabular}



Alive with cancer
0.57
$0.442-0.696$
Beta
de Almeida JR
et al. [26]

Death (cancer death or other 0 death)

\section{Cost (\$)}

\begin{tabular}{lllll}
\hline IMPT & 50,000 & $\begin{array}{l}37,134.7- \\
62,882.3\end{array}$ & Normal & SPHIC \\
\hline IMRT & 12,000 & $\begin{array}{l}10,737.4- \\
13,282.5\end{array}$ & Normal & SYSUCC \\
& & $\begin{array}{l}3,728.8- \\
6,297.3\end{array}$ & Normal & SYSUCC \\
\hline Concurrent chemotherapy & 5,000 & $869.7-1,128.0$ & Normal & SYSUCC \\
\hline Follow-up / year & 1,000 & $1,716.6-$ & Normal & SYSUCC \\
\hline $\begin{array}{l}\text { Treatment for dysphagia / } \\
\text { year }\end{array}$ & 3,000 & $4,273.0$ & & \\
\hline $\begin{array}{l}\text { Treatment for xerostomia / } \\
\text { year }\end{array}$ & 2,000 & $1,362.6-$ & Normal & SYSUCC \\
Palliative therapy / year & 5,000 & $2,641.6$ & & \\
& & $2,411.2-$ & Normal & SYSUCC \\
\hline
\end{tabular}

\section{Markov model set-up}

Cycle length

Number of cycles

Number of cycles

\section{1-year}

$77^{\mathrm{e}}$ - patient

age

$3 \%$

$\mathrm{Cl}$ confidence interval, $P S A$ probabilistic sensitivity analysis, OPC oropharyngeal cancer, IMPT intensity modulated proton therapy, IMRT intensity modulated radiation therapy, OS overall survival, DFS diseasefree survival, NTCP normal tissue complication probability, $Q A L Y$ quality adjusted life year, $\$$ US dollars, SPHIC Shanghai Proton and Heavy Ion Center, SYSUCC Sun Yat-sen University Cancer Center, CEA costeffectiveness analysis

aProbabilistic sensitivity analysis (PSA) was performed to determine $90 \% \mathrm{Cl}$ for model parameters by running over 50,000 iteration trials.

${ }^{\mathrm{b}}$ The utilities and probabilities were tested using beta distribution and the costs were tested using uniform distribution.

${ }^{\mathrm{C}}$ The patient age when preparing for radiotherapy.

${ }^{\mathrm{d}}$ Median age of OPC patients in China. 
${ }^{\mathrm{e}}$ The estimated Chinese life expectancy. For the base case, Markov models were to be cycled 21 times to evaluate the outcomes over a time-period from 1 year after radiotherapy to the end of the estimated Chinese life expectancy.

Table 2 One-way sensitivity analysis identifying cost-effective scenarios of proton beam therapy with different willingness-to-pay thresholds

\begin{tabular}{|c|c|c|c|c|c|}
\hline \multirow[t]{2}{*}{ Parameters } & \multirow{2}{*}{$\begin{array}{l}\text { Base Case Set- } \\
\text { up }\end{array}$} & \multirow{2}{*}{$\begin{array}{l}\text { Expected } \\
\text { Value }\end{array}$} & \multicolumn{3}{|c|}{ Cost-effective Threshold Value } \\
\hline & & & $\begin{array}{l}\$ 30,828 / \\
Q A L Y^{b}\end{array}$ & $\begin{array}{l}\$ 50,000 / \\
\text { QALY }\end{array}$ & $\begin{array}{l}\$ 100,000 / \\
\text { QALY }\end{array}$ \\
\hline NTCP-reduction ${ }^{a}$ & $25 \%$ & Minimum & $57.3 \%$ & $22.6 \%$ & $1.4 \%$ \\
\hline $\begin{array}{l}\text { Patient age (year- } \\
\text { old) }\end{array}$ & 56 & Maximum & 38 & 56 & 68 \\
\hline $\begin{array}{l}\text { The cost of IMPT } \\
(\$)\end{array}$ & 50,000 & Maximum & $37,398.1$ & $51,281.9$ & $87,490.4$ \\
\hline
\end{tabular}

NTCP normal tissue complication probability, IMPT intensity modulated proton radiation therapy, $\$$ US dollars, $Q A L Y$ quality-adjusted life-year

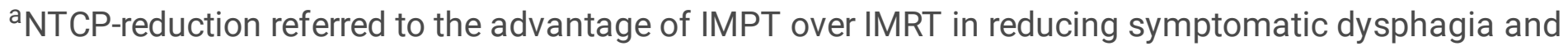
xerostomia, and calculated with the formula: NTCP-reduction (\%) = [(NTCP after IMRT - NTCP after IMPT $)$ / NTCP after IMRT $]$ *100\%.

${ }^{b}$ The willingness-to-pay threshold of China.

\section{Figures}


No Cancer

Alive With Cancer

Death

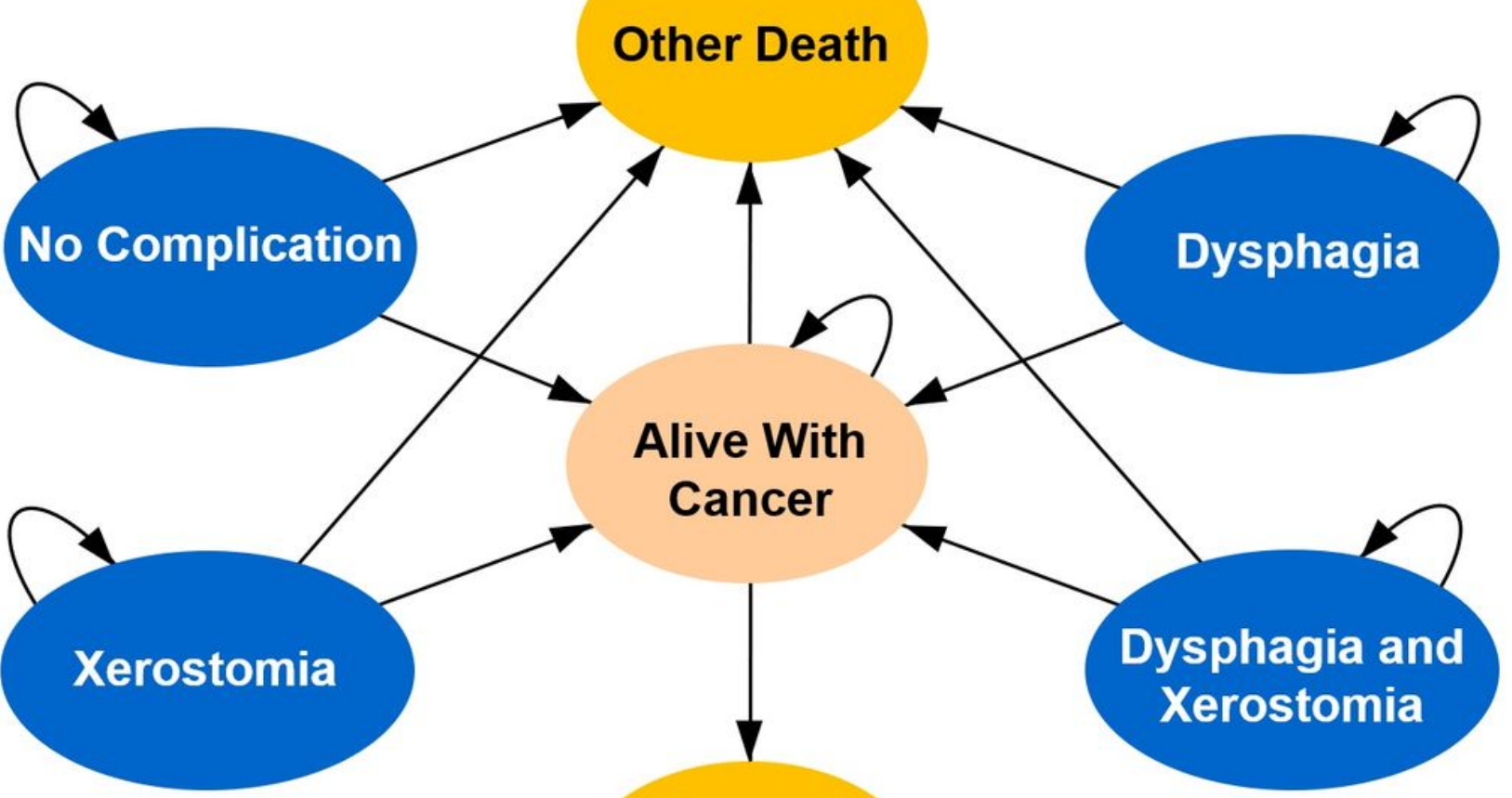

Cancer Death

\section{Figure 1}

Transition states of Markov model Legend: 3 main Markov states of "no cancer", "alive with cancer" and "death" were used to simulate the disease process of oropharyngeal cancer. The state of "no cancer" included four sub-states "dysphagia", "xerostomia", "dysphagia and xerostomia" and "no complication"; and their initial state probabilities were set according to NTCPs. For each cycle, if the patient was in the state of "no cancer", s/he might stay in the state of "no cancer", develop into the state of "alive with cancer" or develop into the state of "death" ("other death"); If the patient was in the state of "alive with cancer", s/he might stay in the state of "alive with cancer" or develop into the state of "death" ("cancer death" or "other death"); If the patient was in the absorbing state "death", the loop operation would be terminated. 1-year cycle length was used, and the Markov model will be cycled from 1 year after radiotherapy until the patient's 77-year-old (Chinese life expectancy). 
List of Influential Parameters Affecting the ICER in Descending Order

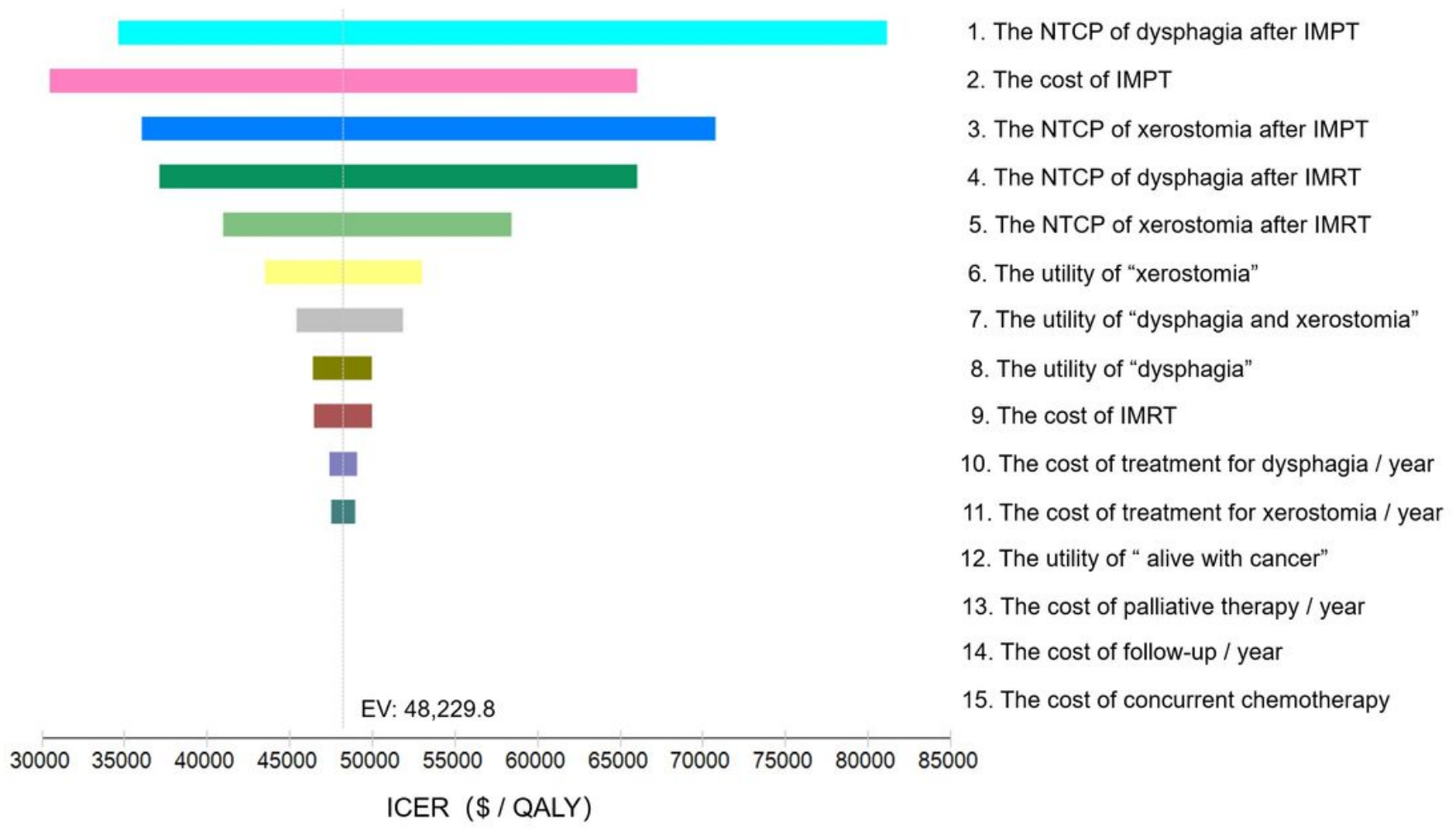

Figure 2

Tornado diagram analyzing of influential parameters affecting the incremental cost-effectiveness ratio Legend: The tornado diagram demonstrated the range of incremental cost-effectiveness ratio (ICER) when varying each parameter individually. Influential parameters were listed in descending order according to their abilities of affecting the ICER over the variation of their $90 \%$ confidence interval. IMPT, intensity modulated proton radiation therapy; IMRT, intensity modulated photon-radiation therapy; NTCP, normal tissue complication probability; EV, expected value; QALY, quality adjusted life year; \$, US dollars. 


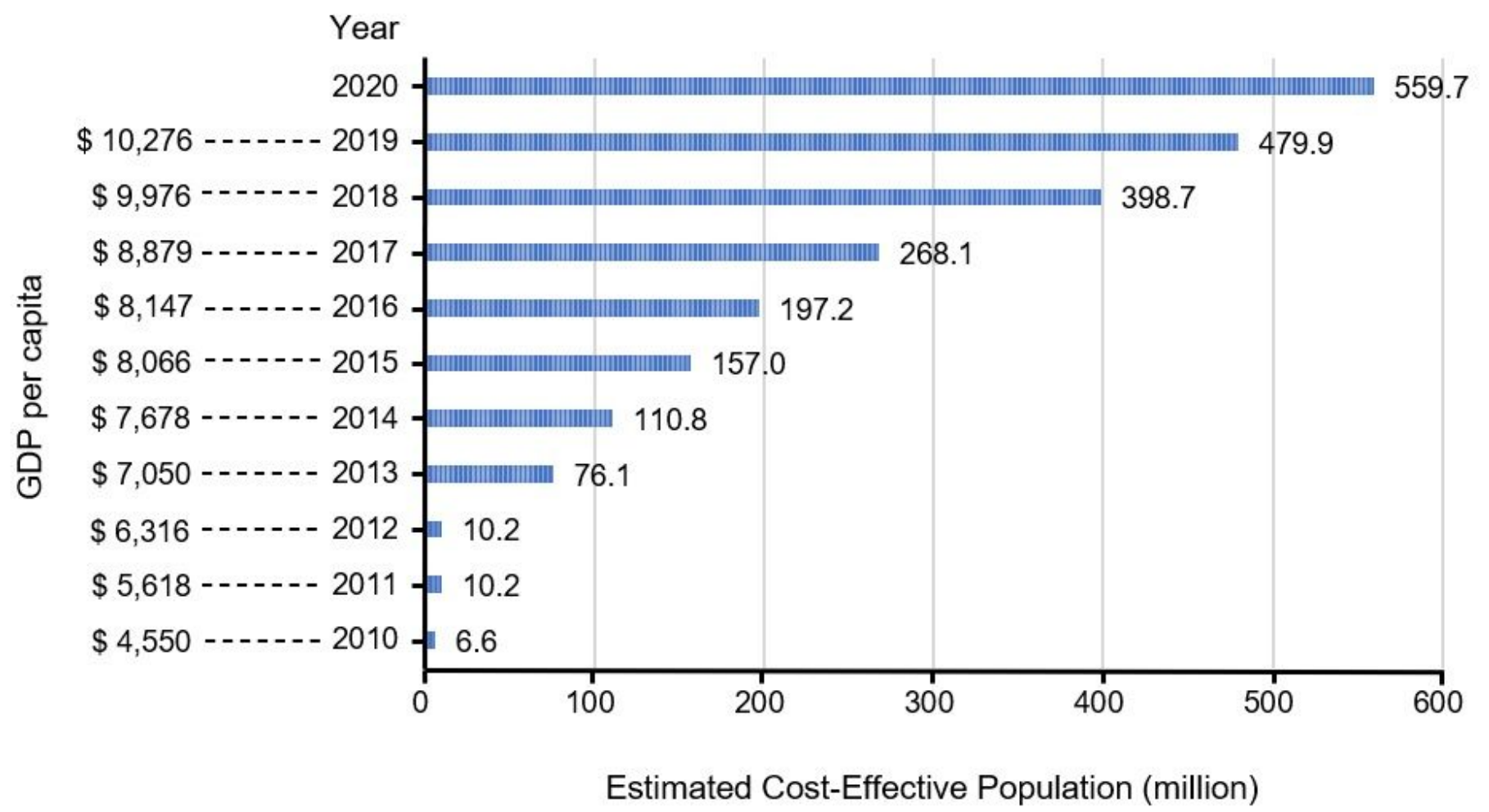

Figure 3

The estimated cost-effective population that benefit from proton beam therapy in China in the past 10 years Legend: The population with a WTP level (3 times the GDP per capita / QALY) beyond the ICER value (\$48,229.8/ QALY) was estimated as cost-effective population in China. \$, US dollars; GDP, gross domestic product; QALY, quality adjusted life year; ICER, incremental cost-effectiveness ratio.

\section{Supplementary Files}

This is a list of supplementary files associated with this preprint. Click to download.

- Additionalfile1FigureS1.tif

- Additionalfile2Figures2.tif

- Additionalfile3FigureS3.tif

- Additionalfile4Tables1.docx

- Additionalfile5Tables2.docx 